\title{
EXCITATION OF OSCILLATIONS - AN UPDATE OF BISON DATA
}

\author{
W. J. CHAPLIN, Y. ELSWORTH, G. R. ISAAK, C. P. MCLEOD AND \\ B. A. MILLER \\ School of Physics \& Astronomy, University of Birmingham \\ Edgbaston, Birmingham B15 2TT UK \\ E-mail: ype@star.sr.bham.ac.uk
}

AND

R. NEW

School of Science \& Mathematics, Sheffield Hallam University

Sheffield, $S 11 \mathrm{WB}$

BiSON is a 6-station, world-wide network of instruments which observe low-degree solar oscillations.

\section{Excitation of oscillations - comparison with theory}

Theory (Goldreich \& Keeley, 1977) predicts that the probability distribution of the mode powers is expected to follow negative exponential statistics under the condition that the interval of time for which the modes are averaged is less than the lifetime of the mode. Simulations (Chaplin et al., 1997) confirm this. The first publication of such an analysis for real data (Elsworth et al., 1995) showed that the oscillations largely follow the predictions with a small excess of the very largest power in the modes. In this paper we extend the previous observations of BiSON data to 80 months of data taken during the declining phase of the solar cycle. The data span the period January 1990 to August 1996. The data have been analysed using Fourier transforms each about $1 / 2$ day in length. The minimum data fill accepted in any one period was 0.7 (Elsworth et al., 1995). Below this fill the data were discarded. The resolution of such short transforms is insufficient to distinguish between the components of the even and odd mode pairs. The mode powers are calculated for mode pairs $\ell=0 \& 2$ and $\ell=1 \& 3$.

Comparison with theoretical predictions show that, as seen previously, there is still an excess of very high excitations which can be seen throughout the 80 months.

\section{Extending the analysis range}

We integrate for less than one lifetime of a mode in order to be able to follow its evolution. At frequencies below about $2.0 \mathrm{mHz}$ the lifetimes of the modes increase (Chaplin et al., 1997) so that the length of the individual sub-set transforms can 
also increase. An indication of the background noise against which the mode must be detected can be found from the measured power between the modes. The signal to noise derived in this way together with the known linewidths indicates that the individual modes can be followed from $2 \mathrm{mHz}$ to $1.6 \mathrm{mHz}$ using 4-day transforms.

\section{References}

Goldreich P., \& Keeley D. A., 1977, ApJ., 211, 934

Chaplin W. J., Elsworth Y., Isaak G. R., McLeod C. P., Miller B. A. and New R., 1997, $M N R A S$, 287, 51

Elsworth Y., Howe R., Isaak G. R., McLeod C. P., Miller B. A. and New R., 1995, GONG 94,51

Chaplin W. J., Elsworth Y., Isaak G. R., McLeod C. P., Miller B. A. and New R., 1997, $M N R A S, 288,623$ 